\title{
ON SOME PROPERTIES OF BANACH OPERATORS
}

\section{A. B. THAHEEM and ABDUL RAHIM KHAN}

(Received 16 November 2000)

\begin{abstract}
A mapping $\alpha$ from a normed space $X$ into itself is called a Banach operator if there is a constant $k$ such that $0 \leq k<1$ and $\left\|\alpha^{2}(x)-\alpha(x)\right\| \leq k\|\alpha(x)-x\|$ for all $x \in X$. In this note we study some properties of Banach operators. Among other results we show that if $\alpha$ is a linear Banach operator on a normed space $X$, then $N(\alpha-1)=N\left((\alpha-1)^{2}\right)$, $N(\alpha-1) \cap R(\alpha-1)=(0)$ and if $X$ is finite dimensional then $X=N(\alpha-1) \oplus R(\alpha-1)$, where $N(\alpha-1)$ and $R(\alpha-1)$ denote the null space and the range space of $(\alpha-1)$, respectively and 1 is the identity mapping on $X$. We also obtain some commutativity results for a pair of bounded linear multiplicative Banach operators on normed algebras.
\end{abstract}

2000 Mathematics Subject Classification. 46C05, 47A10, 47A50, 47H10.

1. Introduction. Let $X$ be a normed space and $\alpha: X \rightarrow X$ a mapping. Following [5], $\alpha$ is said to be a Banach operator if there exists a constant $k$ such that $0 \leq k<1$ and $\left\|\alpha^{2}(x)-\alpha(x)\right\| \leq k\|\alpha(x)-x\|$ for all $x \in X$. Banach operators generalize contractions and play an important role in the fixed point theory; their consideration essentially goes back to Cheney and Goldstein [4] in the study of proximity maps on convex sets (cf. [5] and the references therein). The purpose of this note is to study some further properties of Banach operators. In Section 2, we prove some decomposition results for Banach operators. For instance, we show that if $\alpha$ is a linear Banach operator on a normed space $X$, then $N(\alpha-1)=N\left((\alpha-1)^{2}\right), N(\alpha-1) \cap R(\alpha-1)=(0)$, and if $X$ is finite dimensional, then $X=N(\alpha-1) \oplus R(\alpha-1)$, where $N(\alpha-1)$ and $R(\alpha-1)$ denote the null space of $(\alpha-1)$ and the range space of $(\alpha-1)$, respectively and 1 is the identity mapping on $X$. This result is no longer true when $X$ is infinite dimensional. However, if $\alpha$ is a bounded linear operator on a Hilbert space $H$ such that $\alpha$ and $\alpha^{*}$ have common fixed points, then it is shown that $N(\alpha-1)+R(\alpha-1)$ is dense in $H$. Though this result is not directly related to Banach operators, yet it provides information about decompositions; therefore, it is of independent interest and we include it here. The techniques in the proof of this decomposition theorem lead to an alternate proof of a well-known result on the residual spectrum of a bounded normal operator on $H$.

In Section 3, we study the operator equation $\alpha+c \alpha^{-1}=\beta+c \beta^{-1}$ for a pair of invertible bounded linear Banach operators $\alpha$ and $\beta$ on a normed space $X$ where $c$ is an appropriate real or complex number. We briefly recall that the equation $\alpha+$ $\alpha^{-1}=\beta+\beta^{-1}$ has been extensively studied for a pair of automorphisms $\alpha, \beta$ on von Neumann algebras, $C^{*}$-algebras and rings. Most of the results deal with decomposition of the underlying structures with an additional assumption of commutativity of $\alpha$ and $\beta$. Certain situations are identified when the equation itself implies the commutativity of $\alpha$ and $\beta$. Recently, this equation has been generalized as $a \alpha+$ 
$b \alpha^{-1}=a \beta+b \beta^{-1}$ for linear combinations $a \alpha+b \alpha^{-1}$ of appropriate real or complex numbers $a, b$. If we put $c=b / a$ with $a \neq 0$, then the equation $a \alpha+b \alpha^{-1}=a \beta+b \beta^{-1}$ becomes $\alpha+c \alpha^{-1}=\beta+c \beta^{-1}$ (cf. [3]). Among other results we show here that if $\alpha$ and $\beta$ are invertible bounded linear multiplicative Banach operators on a normed algebra $X$ with identity such that $\alpha(x)+c \alpha^{-1}(x)=\beta(x)+c \beta^{-1}(x)$ for all $x \in X$ and if $\beta$ is inner (i.e., there exists an invertible element $u \in X$ such that $\beta(x)=u x u^{-1}$ for all $x \in X)$, then $\alpha, \beta$ commute. For more on these operator equations we refer to $[1,3,8]$ where further references are given.

2. Decomposition results. Let $X$ be a normed space and $\alpha$ a linear operator on $X$. Denote by $N(\alpha)$, the null space of $\alpha$ and $R(\alpha)$, the range space of $\alpha$. If $X$ is finite dimensional and $N(\alpha)=N\left(\alpha^{2}\right)$ then by the dimension theory of finite-dimensional spaces, $R(\alpha)=R\left(\alpha^{2}\right)$ and $X$ admits the decomposition $X=N(\alpha) \oplus R(\alpha)$. This result may not be true when $X$ is infinite dimensional. In fact, one cannot expect even a weaker result that $N(\alpha)+R(\alpha)$ is dense in $X$. For instance, one can find an injective operator whose range is not dense. However, if $\alpha$ is a bounded linear Banach operator on a normed space $X$, then $N(\alpha-1)=N\left((\alpha-1)^{2}\right), N(\alpha-1) \cap R(\alpha-1)=(0)$, where 1 denotes the identity operator on $X$ and if $X$ is finite dimensional, then $X=N(\alpha-1) \oplus$ $R(\alpha-1)$. These and some other results are proved in this section.

Proposition 2.1. Let $\alpha$ be a linear Banach operator on normed space $X$, then

(i) $N(\alpha-1)=N\left((\alpha-1)^{2}\right)$,

(ii) $N(\alpha-1) \cap R(\alpha-1)=(0)$.

Proof. (i) $N(\alpha-1) \subseteq N\left((\alpha-1)^{2}\right)$ is obvious. Assume $x \in N\left((\alpha-1)^{2}\right)$. Then $(\alpha-1)^{2}(x)=\alpha^{2} x-2 \alpha(x)+x=0$; so $\alpha(x)-x=\alpha^{2}(x)-\alpha(x)$ and since $\alpha$ is a Banach operator, we get $\|\alpha(x)-x\|=\left\|\alpha^{2}(x)-\alpha(x)\right\| \leq k\|\alpha(x)-x\|$. Since $0 \leq k<1$, we get $\|\alpha(x)-x\|=0$; that is, $\alpha(x)=x$ or $x \in N(\alpha-1)$. Thus $N\left((\alpha-1)^{2}\right) \subseteq N(\alpha-1)$ and hence $N(\alpha-1)=N\left((\alpha-1)^{2}\right)$.

(ii) To prove $N(\alpha-1) \cap R(\alpha-1)=(0)$, assume that $y \in N(\alpha-1) \cap R(\alpha-1)$. Then $(\alpha-1)(y)=0$ and $y=(\alpha-1)(x)$ for some $x \in X$ and hence $(\alpha-1)(y)=$ $(\alpha-1)^{2}(x)=0$ so that $x \in N\left((\alpha-1)^{2}\right)=N(\alpha-1)$, (by (i)). Thus $y=(\alpha-1)(x)=0$ and hence $N(\alpha-1) \cap R(\alpha-1)=(0)$.

It is obvious that $R\left((\alpha-1)^{2}\right) \subseteq R(\alpha-1)$. Thus $N\left((\alpha-1)^{2}\right) \cap R\left((\alpha-1)^{2}\right) \subseteq N\left((\alpha-1)^{2}\right) \cap$ $R(\alpha-1)=N(\alpha-1) \cap R(\alpha-1)=(0)$, so that $N\left((\alpha-1)^{2}\right) \cap R\left((\alpha-1)^{2}\right)=(0)$.

If $X$ is finite dimensional then by the dimension theory of finite-dimensional spaces, we can conclude that $R\left((\alpha-1)^{2}\right)=R(\alpha-1)$ and hence by Taylor [7, pages 271-273] $X=N(\alpha-1) \oplus R(\alpha-1)$. Thus we have the following proposition.

Proposition 2.2. Let $\alpha$ be a linear Banach operator on a finite-dimensional normed space $X$, then $X=N(\alpha-1) \oplus R(\alpha-1)$.

For certain operators on Hilbert spaces, we are able to get somewhat general forms of the above result though it would be interesting to obtain a similar result for Banach operators. However, this result is of independent interest in operator theory, so we include it here. 
Proposition 2.3. Let $\alpha$ be a bounded linear operator on a Hilbert space $H$ such that $\alpha$ and $\alpha^{*}$ have common fixed points, then $N(\alpha-1) \cap R(\alpha-1)=(0)$ and $N(\alpha-1)+$ $R(\alpha-1)$ is dense in $H$.

Proof. If $x \in N(\alpha-1) \cap R(\alpha-1)$, then $\alpha(x)=x$. Thus (by assumption) $\alpha^{*}(x)=x$; that is, $x \in N\left(\alpha^{*}-1\right)=N\left((\alpha-1)^{*}\right)=\overline{R(\alpha-1)}{ }^{\perp}$. This implies that $\langle x, x\rangle=0$ and hence $x=0$. To prove the second part, assume that $N(\alpha-1)+R(\alpha-1)$ is not dense in $H$. Then there exists a nonzero continuous linear functional $\phi$ on $H$ such that $\phi$ vanishes on $N(\alpha-1)$ and $R(\alpha-1)$. That $\phi$ vanishes on $R(\alpha-1)$ implies $\phi(\alpha(x)-x)=(\phi \circ \alpha)(x)-\phi(x)=0$ or $(\phi \circ \alpha)(x)=\phi(x)$ for all $x \in H$. By the Riesz-representation theorem, there is a unique $y_{0} \in H$ such that $\phi(x)=\left\langle x, y_{0}\right\rangle=$ $(\phi \circ \alpha)(x)=\left\langle\alpha(x), y_{0}\right\rangle=\left\langle x, \alpha^{*}\left(y_{0}\right)\right\rangle$ for all $x \in H$ and hence $\alpha^{*}\left(y_{0}\right)=y_{0}$. By assumption, $\alpha\left(y_{0}\right)=y_{0}$; that is, $y_{0} \in N(\alpha-1)$ and hence $\phi\left(y_{0}\right)=\left\langle y_{0}, y_{0}\right\rangle=\left\|y_{0}\right\|^{2}=0$; that is, $y_{0}=0$. It follows that $\phi(x)=0$ for all $x \in H$, so $\phi=0$, a contradiction. This completes the proof.

If $\alpha$ is a linear contraction on $H$ then $\alpha$ and $\alpha^{*}$ have common fixed points (cf. [2, page 188]); therefore, we have the following corollary.

COROLLARY 2.4. If $\alpha$ is a linear contraction on a Hilbert space $H$, then $N(\alpha-1) \cap$ $R(\alpha-1)=(0)$ and $N(\alpha-1)+R(\alpha-1)$ is dense in $H$.

If $\alpha$ is a bounded normal operator on $H$, then $\|(\alpha-1)(x)\|=\left\|\left(\alpha^{*}-1\right) x\right\|$ for all $x \in H$ and hence $\alpha$ and $\alpha^{*}$ have common fixed points; therefore, we have the following corollary.

COROLlary 2.5. If $\alpha$ is a bounded normal operator on a Hilbert space $H$, then $N(\alpha-1) \cap R(\alpha-1)=(0)$ and $N(\alpha-1)+R(\alpha-1)$ is dense in $H$.

It is well known that the residual spectrum of a bounded normal operator on a Hilbert space $H$ is empty (cf. [6, page 479]). The techniques in the proof of the above proposition can be applied to obtain an alternate proof of this result.

Proposition 2.6. The residual spectrum $R_{\sigma}(\alpha)$ of a bounded normal operator $\alpha$ on a Hilbert space $H$ is empty.

Proof. Let $\lambda \in R_{\sigma}(\alpha)$. Then $N(\alpha-\lambda)=(0)$ and $\overline{R(\alpha-\lambda)} \neq H$. Let $\phi$ be a nonzero continuous linear functional on $H$ such that $\phi$ vanishes on $\overline{R(\alpha-\lambda)}$. By the Rieszrepresentation theorem, there is a unique $y_{0} \in H$ such that $\phi(x)=\left\langle x, y_{0}\right\rangle$ for all $x \in H$. Now $\phi$ vanishes on $\overline{R(\alpha-\lambda)}$; therefore, $\phi(\alpha(x))=(\phi \circ \alpha)(x)=\lambda \phi(x)$ for all $x \in H$ and hence $\phi(\alpha(x))=\lambda \phi(x)=\lambda\left\langle x, y_{0}\right\rangle=\left\langle x, \bar{\lambda} y_{0}\right\rangle$. Also, $\phi(\alpha(x))=\left\langle\alpha(x), y_{0}\right\rangle=\langle x$, $\left.\alpha^{*}\left(y_{0}\right)\right\rangle$, so that $\left\langle x, \bar{\lambda} y_{0}\right\rangle=\left\langle x, \alpha^{*}\left(y_{0}\right)\right\rangle$ for all $x \in H$ and hence $\alpha^{*}\left(y_{0}\right)=\bar{\lambda} y_{0}$. Since $\alpha$ is normal, we get $\alpha\left(y_{0}\right)=\lambda y_{0}$ and hence $y_{0} \in N(\alpha-\lambda)$ and consequently $y_{0}=0$. Thus $\phi=0$, a contradiction and hence $\sigma_{R}(\alpha)$ is empty. This completes the proof.

3. Commutativity results. In this section, we mainly obtain some commutativity results for a pair of invertible bounded linear Banach operators $\alpha, \beta$ satisfying the equation $\alpha+c \alpha^{-1}=\beta+c \beta^{-1}$ on normed algebras. We begin with the following basic result. 
Proposition 3.1. Let $\alpha$ be an invertible linear Banach operator on a normed space $X$ and $x_{0} \in X$ such that $\alpha\left(x_{0}\right)+c \alpha^{-1}\left(x_{0}\right)=(1+c) x_{0}$, where $c$ is a real or complex number with $|c| \geq 1$, then $x_{0}$ is a fixed point of $\alpha$.

Proof. The equation implies that $\alpha^{2}\left(x_{0}\right)+c x_{0}=(1+c) \alpha\left(x_{0}\right)$ and hence $c\left(\alpha\left(x_{0}\right)-x_{0}\right)$ $=\alpha^{2}\left(x_{0}\right)-\alpha\left(x_{0}\right)$. Since $\alpha$ is a Banach operator, therefore there exists a constant $k$ with $0 \leq k<1$ such that $|c|\left\|\alpha\left(x_{0}\right)-x_{0}\right\|=\left\|\alpha^{2}\left(x_{0}\right)-\alpha\left(x_{0}\right)\right\| \leq k\left\|\alpha\left(x_{0}\right)-x_{0}\right\|$ and hence $\left\|\alpha\left(x_{0}\right)-x_{0}\right\| \leq(k /|c|)\left\|\alpha\left(x_{0}\right)-x_{0}\right\| \leq k\left\|\alpha\left(x_{0}\right)-x_{0}\right\|$ and since $0 \leq k<1$, we obtain $\alpha\left(x_{0}\right)-x_{0}=0$ or $\alpha\left(x_{0}\right)=x_{0}$.

The following proposition shows that under certain situations the equation $\alpha+$ $c \alpha^{-1}=\beta+c \beta^{-1}$ implies the commutativity of $\alpha, \beta$.

Proposition 3.2. Let $\alpha, \beta$ be invertible linear multiplicative Banach operators on a normed algebra $X$ with identity such that $\alpha(x)+c \alpha^{-1}(x)=\beta(x)+c \beta^{-1}(x)$ for all $x \in X$, where $c$ is a real or complex number with $|c| \geq 1$. If $\beta$ is inner, then $\alpha, \beta$ commute.

Proof. Assume that $\beta(x)=u x u^{-1}$ for all $x \in X, u \in X$. Putting $x=u$ in the equation, we get $\alpha(u)+c \alpha^{-1}(u)=(1+c) u$ and by Proposition 3.1, $\alpha(u)=u$, and hence $(\beta \alpha)(x)=\beta(\alpha(x))=u \alpha(x) u^{-1}=\alpha(u) \alpha(x) \alpha\left(u^{-1}\right)=\alpha\left(u x u^{-1}\right)=\alpha(\beta(x))=$ $(\alpha \beta)(x)$ for all $x \in X$. This proves that $\alpha, \beta$ commute.

Proposition 3.3. Let $\alpha, \beta$ be invertible bounded linear multiplicative Banach operators on a normed algebra $X$ with identity such that $\alpha(x)+c \alpha^{-1}(x)=\beta(x)+c \beta^{-1}(x)$ for all $x \in X,\|\alpha-1\|<1,\|\beta-1\|<1$, where $c$ is a real or complex number with $|c|>4$ and $\beta$ is inner. Then $\alpha=\beta$.

Proof. By Proposition 3.2, $\alpha, \beta$ commute. The equation $\alpha(x)+c \alpha^{-1}(x)=\beta(x)+$ $c \beta^{-1}(x)$ together with the commutativity of $\alpha, \beta$ implies $(\alpha \beta-c)\left(\beta^{-1}-\alpha^{-1}\right)(x)=0$. Put $\left(\beta^{-1}-\alpha^{-1}\right)(x)=y$. Then $(\alpha \beta-c)(y)=0$ or $\alpha \beta(y)=c y$, so that $c y-\beta y=\alpha \beta y-$ $\beta y=(\alpha-1) \beta(y)$ and hence $\|c y-\beta(y)\|=\|(\alpha-1) \beta(y)\| \leq\|\alpha-1\|\|\beta(y)\|<\|\beta(y)\|$. That is, $|\|c y\|-\|\beta(y)\||<\|\beta(y)\|$. This implies that $|c|\|y\|<2\|\beta(y)\| \leq 2\|\beta\|\|y\|$. Since $\|\beta-1\|<1$, we get $|\|\beta\|-1| \leq\|\beta-1\|<1$ and hence $\|\beta\|<2$ so that $|c|\|y\|<$ $4\|y\|$. If $y \neq 0$, then we get $|c|<4$, a contradiction; therefore $y=0$ and hence $\beta^{-1}(x)-$ $\alpha^{-1}(x)=0$ for all $x \in X$. Since $\alpha, \beta$ commute and $\alpha \beta$ is linear, we get $\alpha(x)-\beta(x)=0$; that is, $\alpha(x)=\beta(x)$ for all $x \in X$ and hence $\alpha=\beta$. This completes the proof.

ACKNOWLEDGEMENT. The authors gratefully acknowledge the support provided by King Fahd University of Petroleum and Minerals during this research.

\section{REFERENCES}

[1] M. Awami and A. B. Thaheem, A short proof of a decomposition theorem of a von Neumann algebra, Proc. Amer. Math. Soc. 92 (1984), no. 1, 81-82. MR 85i:46082. Zbl 568.46046.

[2] S. K. Berberian, Lectures in Functional Analysis and Operator Theory, Springer-Verlag, New York, 1974. MR 54\#5775. Zbl 296.46002.

[3] F. S. Cater and A. B. Thaheem, On certain equations of automorphisms of $C^{*}$-algebras, preprint. 
[4] W. Cheney and A. A. Goldstein, Proximity maps for convex sets, Proc. Amer. Math. Soc. 10 (1959), 448-450. MR 21\#3755. Zbl 092.11403.

[5] S. A. Naimpally, K. L. Singh, and J. H. M. Whitfield, Fixed points and nonexpansive retracts in locally convex spaces, Fund. Math. 120 (1984), no. 1, 63-75. MR 85m:47062. Zbl 561.47047.

[6] C. Swartz, An Introduction to Functional Analysis, Marcel Dekker, New York, 1992. MR 93c:46002. Zbl 751.46002.

[7] A. E. Taylor, Introduction to Functional Analysis, John Wiley and Sons, New York, 1958. MR 20\#5411. Zbl 081.10202.

[8] A. B. Thaheem, On certain decompositional properties of von Neumann algebras, Glasgow Math. J. 29 (1987), no. 2, 177-179. MR 88i:46085. Zbl 634.46050.

A. B. Thaheem: Department of Mathematical Sciences, King Fahd University of Petroleum AND Minerals, Dhahran 31261, SAudi Arabia

E-mail address: athaheem@kfupm.edu.sa

Abdul Rahim Khan: Department of Mathematical Sciences, King Fahd University of Petroleum AND Minerals, DHaHran 31261, SAUdi Arabia

E-mail address: arahim@kfupm. edu.sa 


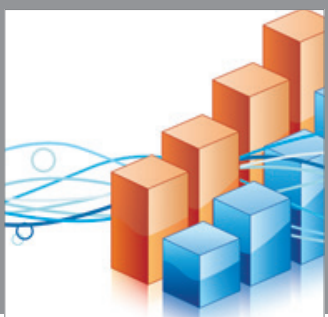

Advances in

Operations Research

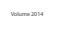

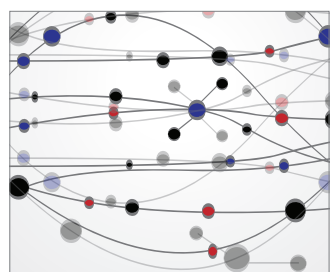

\section{The Scientific} World Journal
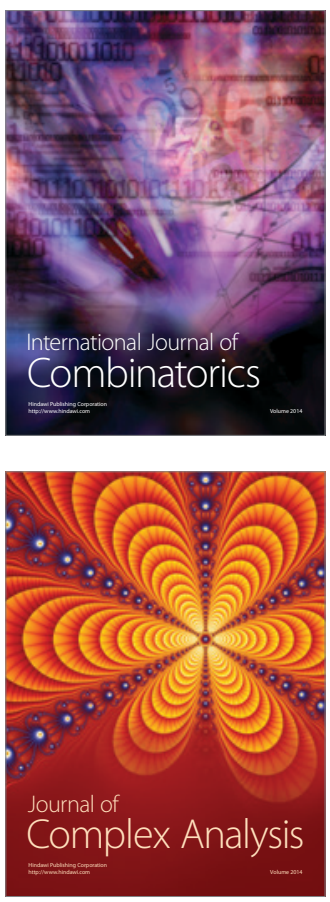

International Journal of

Mathematics and

Mathematical

Sciences
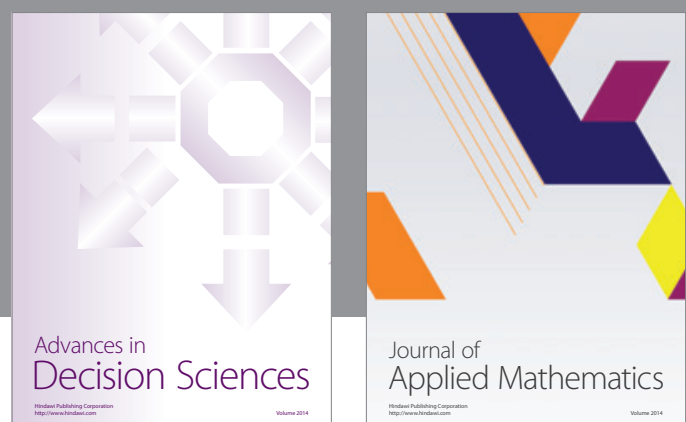

Journal of

Applied Mathematics
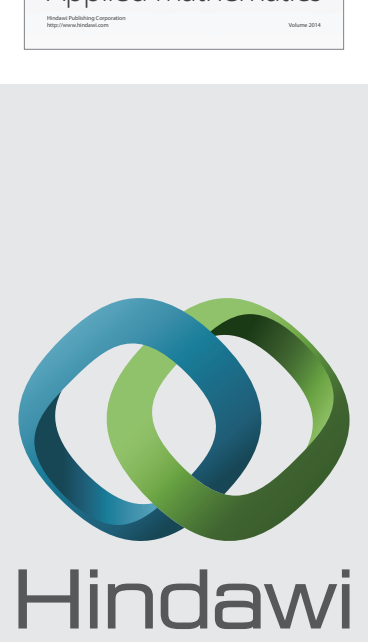

Submit your manuscripts at http://www.hindawi.com
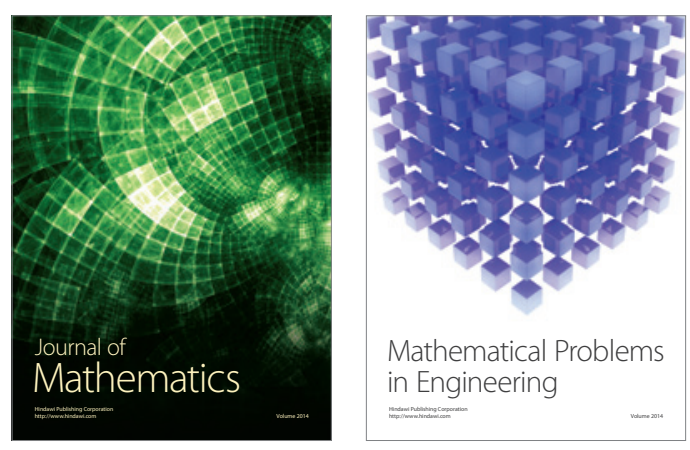

Mathematical Problems in Engineering
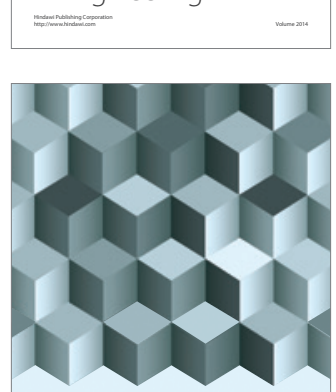

Journal of

Function Spaces


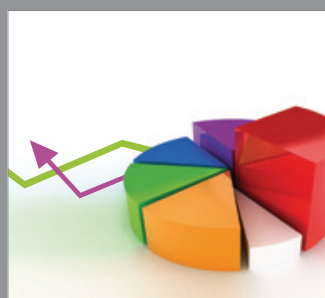

ournal of

Probability and Statistics

Promensencen
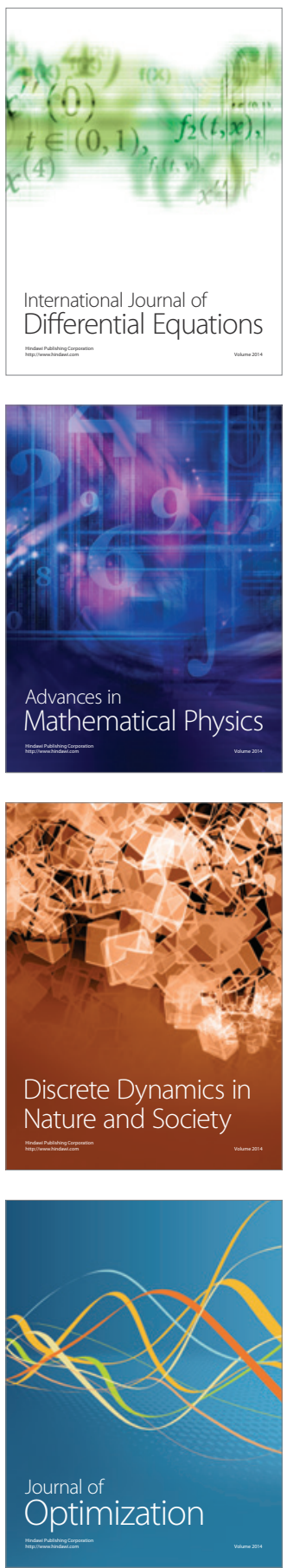